Conclusion: We showed that SLE patients who had constitutional findings and LAP could be the presenting feature and may confer a significant diagnostic challenge in which the patients might require invasive procedures and advanced imaging modalities. PET/CT revealed reactive changes in lymph nodes in all patients but lymph node biopsy revealed atypical changes in some patients suggestive of viral infections. On the other hand, LAP patients with constitutional symptoms represents a severe phonotype of the SLE as there were significant renal and thrombotic disease.

Disclosure of Interests: None declared

DOI: 10.1136/annrheumdis-2021-eular.2588

\section{POS0751 COMORBIDITY AND LONG-TERM OUTCOME IN PATIENTS WITH CONGENITAL HEART BLOCK: PRELIMINARY DATA OF THE ITALIAN REGISTRY ON THE IMMUNE-MEDIATED CONGENITAL HEART BLOCK}

M. Fredi ${ }^{1}$, G. Rizzo ${ }^{2}$, L. Andreoli ${ }^{1}$, B. Bacco ${ }^{3}$, T. Bertero $^{3}$, A. Bortoluzzi ${ }^{4}$, F. Ceccarelli ${ }^{5}$, R. Cimaz ${ }^{6}$, P. Conigliaro ${ }^{7}$, F. Corradi $^{3}$, S. De Vita ${ }^{8}$, E. DI Poi ${ }^{8}$, E. Elefante ${ }^{9}$, G. Emmi ${ }^{10}$, M. Gerosa ${ }^{11}$, M. Govoni ${ }^{4}$, A. Hoxha ${ }^{12}$, A. Lojacono ${ }^{13}$, E. Marrani ${ }^{14}$, L. Marozio ${ }^{15}$, A. Mathieu ${ }^{16}$, M. Mosca $^{9}$, P. Melissa ${ }^{4}$, C. Picchi ${ }^{17}$, M. Piga ${ }^{16}$, R. Priori ${ }^{5}$, V. Ramoni ${ }^{18}$, A. Ruffatti ${ }^{19}$, G. Simonini ${ }^{14}$, C. Tani $^{9}$, M. Tonello ${ }^{19}$, L. Trespidi ${ }^{20}$, M. L. Urban ${ }^{10}$, M. Vezzoli ${ }^{21}$, S. Zatti ${ }^{22}$, S. Calza ${ }^{21}$, A. Brucato ${ }^{23}$, F. Franceschini ${ }^{1}$, A. Tincani ${ }^{1}{ }^{1}$ UO Reumatologia e Immunologia Clinica, Dipartimento Scienze Cliniche e Sperimentali, ASST Spedali Civili e Università degli Studi di Brescia, Brescia, Italy; ${ }^{2}$ University of Brescia, Brescia, Italy; ${ }^{3}$ S.S.d.D.U. Immunologia, Allergologia, A.O. Ordine Maurziano di Torino, Torino, Italy; ${ }^{4} \mathrm{UO}$ e Sezione di Reumatologia, Dipartimento di Scienze Mediche, Universita' degli Studi di Ferrara, Ferrara, Italy; ${ }^{5}$ UO Complessa, Reumatologia, Policlinico Umberto I- University La Sapienza, Roma, Italy; ${ }^{6}$ Department of Clinical Sciences and Community Health, University of Milan, Milano, Italy; ${ }^{7}$ Rheumatology, Allergology and Clinical Immunology, Department of Systems Medicine, University of Rome Tor Vergata, Roma, Italy; ${ }^{8}$ Clinica di Reumatologia, Azienda Sanitaria Universitaria Integrata di Udine, Udine, Italy; ${ }^{9}$ UO Reumatologia, Dipartimento di Medicina Clinica e Sperimentale, Universita' di Pisa, Pisa, Italy; ${ }^{10}$ Department of Experimental and Clinical Medicine, University of Florence, Florence, Italy; ${ }^{11}$ Istituto Ortopedico Gaetano Pini, University of Milan, Milan, Italy; ${ }^{12}$ Internal Medicine Unit, Department of Medicine, San Bortolo Hospital, Vicenza, Italy; ${ }^{13}$ Ostetricia e Ginecologia, Ospedale di Desenzano, ASST Garda, Desenzano del Garda, Italy; ${ }^{14}$ Servizio di Reumatologia Pediatrica, Azienda Ospedaliero-Universitaria "Anna Meyer," Firenze, Italy; ${ }^{15}$ Ginecologia e Ostetricia 1, Dipartimento di Scienze Chirurgiche, Università di Torino, Turin, Italy; ${ }^{16}$ Cattedra e Struttura Complessa di Reumatologia, Universita' degli Studi e AOU di Cagliari, Cagliari, Italy; ${ }^{17}$ Medicina Interna, ASST Fatebenefratelli, Milano, Italy; ${ }^{18}$ Rheumatology, IRCCS Policlinico San Matteo Foundation, University of Pavia, Pavia, Italy; ${ }^{19}$ Unità di Reumatologia, Dipartimento di Medicina, Università di Padova, Padova, Italy; ${ }^{20}$ Dipartimento per la Salute della Donna, Bambino e Neonato, Fondazione Ospedale Maggiore, Milan, Italy; ${ }^{21}$ Unit of Biostatistics, Biomathematics, and Bioinformatics, Department of Molecular and Translational Medicine, University of Brescia, Brescia, Italy; ${ }^{22}$ Department of Obstetrics and Gynecology, ASST Spedali Civili and University, Brescia, Italy; ${ }^{23}$ Dipartimento di Scienze Biomediche e Cliniche "Sacco", Università degli Studi di Milano, Milan, Italy

Background: Congenital heart block $(\mathrm{CHB})$ is due to placental transfer of maternal anti-Ro/SSA autoantibodies to the fetus. The prevalence of $\mathrm{CHB}$ has been estimated as $1-2 \%$ in anti-Ro/SSA women while the recurrence rate is $16-19 \%$ (1). This condition is associated with a high rate of fetal/neonatal mortality and most of the cases requires pacemaker (PM) pacing. Given the rarity of $\mathrm{CHB}$, limited data are available regarding the long-term follow-up of the offspring other than the cardiovascular complications.

Objectives: The results of the Italian Registry of the autoimmune congenital heart block were recently described (2). A peculiarity of this cohort was that most of the mothers had an established diagnosis of systemic autoimmune disease at $\mathrm{CHB}$ detection, in contrast with other registries where $\mathrm{CHB}$ was mostly incidentally detected in healthy women. Here we report an update, with the preliminary data regarding the long-term outcome of patients with $\mathrm{CHB}$, their unaffected siblings and health controls born from mothers positive for Ro/SSA.

Methods: Data regarding demography, treatment, maternal, neonatal outcome, and follow-up were collected through an online electronic datasheet. A dedicated questionnaire was created with the aim to investigate general health, cardiovascular follow-up, and frequency of autoimmune diseases.

Results: One-hundred and five cases of $\mathrm{CHB}$ in 99 patients were included from 1969 to December 2020. CHB was mostly detected in utero (97 cases, 92.3\%) with 8 neonatal cases. Third degree CHB occurred in 71 cases $(67.6 \%)$. Child mortality was observed in $29(27.6 \%)$ cases: 20 in utero, 7 during neonatal period and 2 during childhood. Overall, a PM was implanted in 54 out of the 85 live births $(63.5 \%)$. Then, our cohort was divided into 2 subgroups: pregnancy that occurred before $(\mathrm{N}=61)$ and after $2010(\mathrm{~N}=44)$ with the aim to evaluate possible differences among the subgroups. Whereas mortality, PM, CHB degree were similar, $\mathrm{CHB}$ more frequently occurred in the last 10 years among Ro/SSA asymptomatic car riers than in the group of pregnancies before 2010 (53.6\% vs $32.8 \%, p=0.038)$ Questionnaires from 14 surviving CHB cases, 8 unaffected siblings 12 controls born from mothers Ro/SSA positive were collected. Among CHB cases, 6 were males and 8 females, median age 12 years (range 6-28). All presented a third degree $\mathrm{CHB}, 10$ required a neonatal $\mathrm{PM}$ pacing and one had an implantable ECG recorder. PM was substituted at least once in 9 patients, the oldest patient had to change it four times. No dilated cardiomyopathy occurred and most of the patients maintain an annual follow-up. Two cases of autoimmune diseases were registered among $\mathrm{CHB}$ cases, one idiopathic juvenile arthritis and one Cogan's vasculitis, both born from mothers with Sjogren Syndrome. Four cases of neurodevelopmental disorders occurred: three cases of learning disabilities (one in each group) and one case of speech disorder in the sibling group. In addition, a $\mathrm{CHB}$ case presented a stress disorder linked to frequent hospitalizations.

Conclusion: This registry is an ongoing project aiming at collecting all Italian $\mathrm{CHB}$. Moreover, here we reported the preliminary data concerning the evaluation of long-term follow-up of CHB patients. Our data, even if need to be confirmed in larger cohort, seems reassuring: no differences were reported comparing $\mathrm{CHB}$ patients with unaffected siblings or controls.

\section{REFERENCES:}

[1] Brito-Zéron et al. Nat Rev Rheumatol 2015;11:301-312.

[2] Fredi M et al. Front Cardiovasc Med. 2019 Feb 28;6:11.

Disclosure of Interests: None declared

DOI: 10.1136/annrheumdis-2021-eular.2697

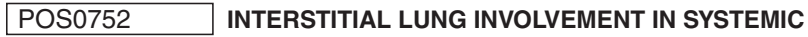 LUPUS ERYTHEMATOSUS: RELEVANT CLINICAL CHARACTERISTICS. OUR EXPERIENCE IN A THIRD LEVEL HOSPITAL}

1. González Fernández ${ }^{1}$, C. Moriano ${ }^{1}$, E. Diez Álvarez ${ }^{1}$, X. E. Larco Rojas ${ }^{1}$, A. López Robles ${ }^{1}$, M. E. Vallejo Pascual ${ }^{2}$, M. T. Perez Sandoval ${ }^{1} .{ }^{1}$ Complejo Asistencial Universitario de León, Reumatología, León, Spain; ${ }^{2}$ Universidad de León, Facultad de CC. EE y Empresariales, León, Spain

Background: Lung involvement ${ }^{1}$ in Systemic Lupus Erythematosus (SLE) is heterogeneous, with the pleura being more frequently affected. Associated diffuse interstitial lung disease (ILD) is rare (3-13\%) with difficult diagnosis and therapeutic management.

Objectives: Assess the main clinical-epidemiological characteristics of patients diagnosed with SLE with ILD in a third level hospital and analyze the possible relationship between them.

Methods: Descriptive observational study of patients diagnosed with SLE (SLICC criteria) with ILD in our hospital between 1973 and 2020. The following clinical-epidemiological characteristics were evaluated such as sex, mean age at diagnosis, presence of cardiovascular risk factors (CVRF), smoking, baseline cardiac and respiratory comorbidities, laboratory markers (autoimmunity, CRP and vitamin D), baseline SLEDAI, manifestations of SLE, interstitial pattern on $\mathrm{HRCT}$, correlation with conventional radiology $(\mathrm{Rx})$, respiratory function tests, clinical and physical examination at diagnosis.

Results: 455 patients diagnosed with SLE were included, of whom 20 had ILD $(4.4 \%) .65 \%$ were women with a mean age at diagnosis of $63 \pm 16.23$ years. $30 \%$ presented ILD as the first clinical data for the diagnosis of SLE. $30 \%$ had jobs with exposure to chemicals. $25 \%$ were ex-smokers and $10 \%$ were active smokers. $10 \%$ had associated CVRF, highlighting $\mathrm{HT}$ (35\%). 30\% had valve disease on echocardiography such as mitral regurgitation (30\%) and tricuspid regurgitation (20\%). Only $10 \%$ had associated bronchopathy. $35 \%$ and $25 \%$ associated respectively, pleural serositis and secondary Sjogren's syndrome. Arthritis and skin involvement were documented in $60 \%$ for both clinical domains. $40 \%$ had SLEDAI> 6

At the diagnosis of ILD, $90 \%$ had symptoms such as dyspnea $(75 \%)$ and cough $(60 \%) .80 \%$ had crackles on examination. $85 \%$ had alterations in the X-ray and $100 \%$ in the CT scan with the NINE patterns as the main one $(65 \%)$ with progression at 2 years despite treatment in $25 \%$. Spirometry differentiated an obstructive pattern (25\%), restrictive $(20 \%)$ and normal $(55 \%)$ with a tendency to stability at one year (40\%) with the prescribed treatment. $90 \%$ had a decrease in diffusion, the majority being mild (67\%). Analytically, $67 \%$ had elevated levels of CRP and hypovitaminosis D; $40 \%$ elevated DNA titers, 50\% hypocomplementemia and $60 \%$ ENAS positive, highlighting Ro (45\%); La (15\%) and RNP (15\%). Leukopenia and thrombopenia (30\% and $10 \%$ respectively) were also observed.

In our study, we did not find any statistical significance between the variables analyzed, due to the small sample size.

Conclusion: 1)ILD is an infrequent manifestation in SLE, it usually associates respiratory symptoms and pulmonary functional repercussions with the diagnosis. The most characteristic radiological pattern is the NINE. 